\title{
Coherent Collisions between Bose-Einstein Condensates
}

\author{
J. M. Vogels, J. K. Chin, and W. Ketterlef \\ Department of Physics, MIT-Harvard Center for Ultracold Atoms, \\ and Research Laboratory of Electronics, Massachusetts Institute of Technology, Cambridge, MA 02139
}

\begin{abstract}
We study the non-degenerate parametric amplifier for matter waves, implemented by colliding two Bose-Einstein condensates. The coherence of the amplified waves is shown by observing high contrast interference with a reference wave and by reversing the amplification process. Since our experiments also place limits on all known sources of decoherence, we infer that relative number squeezing is most likely present between the amplified modes. Finally, we suggest that reversal of the amplification process may be used to detect relative number squeezing without requiring single-particle detection.
\end{abstract}

PACS numbers: 03.75.Fi, 34.50.-s

Quantum entanglement has always attracted great interest as it is one of the key differences between quantum and classical physics. Since it can be exploited for various novel applications like quantum computation and precision measurements, there has been a continuing effort to engineer ever more robust quantum correlated states. Recently, four-wave mixing using Bose-Einstein condensates (BEC's) has been proposed as a method for creating two correlated matter waves [1]. In that experiment, two source waves and a third seed wave were used to produce a fourth wave in the conjugate momentum state, resulting in an amplified seed-conjugate wave pair. For large amplification, the relative number fluctuations between the amplified waves can become significantly reduced, and this was recently achieved [2]. In optics, this is known as non-degenerate parametric amplification [3], where it is also used for generating similarly squeezed photon beams. Once created, such two-mode number squeezed states could be used for Heisenberg-limited interferometry where phase shifts up to $1 / N$ accuracy could be measured [4, 5]. As the occupation number $N$ of the states increases, the advantage gained over the standard shot noise limit of $1 / \sqrt{N}$ becomes quite significant.

Entanglement between the generated matter waves is possible only if the collisional four-wave mixing process is coherent. While the coherence of collisions has been observed before in mean field effects [6, [7], it has not been demonstrated for the more common case of momentum changing elastic collisions. By itself, four wave mixing does not require or guarantee coherence, since it will still occur for condensates with short coherence lengths [8]. Moreover, even though previous four wave mixing experiments 11, 2] utilized two coherent condensates, they did not explicitly show that coherence was maintained throughout the collisional process.

In this paper, we demonstrate the coherence of elastic collisions by observing high contrast interference between the conjugate wave and a reference wave. Due to the strong coherence retained, we also observe a reversal of the amplification process. The success of these two experiments places an upper bound on the decoherence effects in our experiments, providing evidence that a significant amount of relative number squeezing is present in the pair-correlated beams. The reversibility of the amplification process further suggests a possible way of directly detecting the two-mode number squeezing and entanglement between the two correlated states, without requiring sub-shotnoise detection.

In the Heisenberg picture, the evolution of the annihilation operators $a_{3}, a_{4}$ of a mode pair that is parametrically amplified by the source modes $a_{1}, a_{2}$ is given by

$$
\begin{aligned}
\frac{d a_{3}}{d t} & =-\frac{8 \pi \hbar a}{V m} i a_{4}^{\dagger} a_{1} a_{2}-i \frac{1}{2 \hbar} \Delta \varepsilon a_{3} \\
\frac{d a_{4}}{d t} & =-\frac{8 \pi \hbar a}{V m} i a_{3}^{\dagger} a_{1} a_{2}-i \frac{1}{2 \hbar} \Delta \varepsilon a_{4}
\end{aligned}
$$

where $V$ is the volume, $m$ is the mass, $a$ is the s-wave scattering length and $\Delta \varepsilon$ is the energy mismatch between the source modes and the amplified modes [2]. The solution to these equations for highly occupied source modes is an exponential growth with a rate constant $\eta=\sqrt{\left(\frac{2 \bar{\mu}}{\hbar}\right)^{2}-\left(\frac{\Delta \varepsilon}{2 \hbar}\right)^{2}}$ where $\bar{\mu}$ is the geometric mean of the chemical potentials of the two source waves. In our experiment, the multiple waves used were generated from a single BEC using two-photon stimulated optical transitions [9]. The evolution of annihilation operators $a, b$ for modes which are coupled by such a Bragg transition is given by

$$
\begin{aligned}
& \frac{d a}{d t}=-\Omega i b \\
& \frac{d b}{d t}=-\Omega^{*} i a
\end{aligned}
$$

where $\Omega$ is the coupling strength. In both the Bragg and amplification process, the generated wave incurs an overall phase shift of $90^{\circ}$ relative to its source, a detail which becomes important for the interference experiment.

The experimental setup was almost identical to the one used in [2]. Sodium condensates of about 13 million atoms each were created in a cylindrical magnetic 
trap with an axial frequency of $20 \mathrm{~Hz}$ and a radial frequency of $40 \mathrm{~Hz}$. They had a Thomas-Fermi radius of $30 \mu \mathrm{m}$ radially and $60 \mu \mathrm{m}$ axially, a chemical potential of $h \times 1.8 \mathrm{kHz}$ and a speed of sound of $6 \mathrm{~mm} / \mathrm{s}$. From an initial condensate $\mathbf{k}_{1}$, a small seed $\mathbf{k}_{3}$ with a velocity of $14 \mathrm{~mm} / \mathrm{s}$ was generated using a $20 \mu \mathrm{s}$ Bragg (seed) pulse (see Fig. 1). In the next $40 \mu \mathrm{s}$, a second source wave $\mathbf{k}_{2}$ with a velocity of $20 \mathrm{~mm} / \mathrm{s}$ was split off from $\mathbf{k}_{2}$ using a $\pi / 2$ pulse. All Bragg beams in our experiment were detuned from the sodium $\mathrm{D}_{2}$ line by $40 \mathrm{GHz}$ to avoid spontaneous Rayleigh scattering. Subsequent collisions between $\mathbf{k}_{1}$ and $\mathbf{k}_{2}$ acted as a parametric amplifier for the seed wave $\mathbf{k}_{3}$, creating a fourth conjugate wave $\mathbf{k}_{4}$ in the momentum conserving state. Both $\mathbf{k}_{3}$ and $\mathbf{k}_{4}$ modes then grew exponentially with an expected rate of $17 \mathrm{~ms}^{-1}$ on average accross the condensate. At the same time, the parametric amplifier also enhanced spontaneous scattering into initially unseeded modes, giving rise to the collisional halo seen in Fig. 1 [10]. The resulting loss of atoms from the source waves limited the four wave mixing process to $400 \mu \mathrm{s}$.

After $80 \mu$ s of amplification, we probed the phase coherence of the conjugate wave $\mathbf{k}_{4}$ by interfering it with a reference wave. For optimal interference, the reference wave needed to have a well defined relative phase and be identical in size and momentum to $\mathbf{k}_{4}$. The reference wave was outcoupled from $\mathbf{k}_{2}$ using another $20 \mu \mathrm{s}$ Bragg (seed) pulse. By changing the phase of one of the Bragg beams, the phase of the reference wave relative to $\mathbf{k}_{4}$ was varied. The resulting occupation of the conjugate momentum state $\mathbf{k}_{4}$ was monitored by selectively transferring a fixed fraction of atoms in $\mathbf{k}_{4}$ to a detection state $\mathbf{k}_{4}^{\prime}$ (see Fig. 1). For this, a $40 \mu$ s Bragg pulse with large momentum transfer $(2 \hbar k)$ was used. This ensured that the $\mathbf{k}_{4}^{\prime}$ atoms were well-separated during ballistic expansion and their number was easily determined. Direct number measurement of the mode $\mathbf{k}_{4}$ was not possible since the amplification process continued during ballistic expansion.

The interference fringe obtained is plotted in Fig. 2. By fitting a sinusoidal curve to the raw data, we obtained a fringe contrast of $68 \pm 11 \%$. This proves that the conjugate wave maintains the phase relation established during amplification and is in a single quantum state. In addition, we expected destructive interference to take place at $90^{\circ}$ due to the accumulation of all the $90^{\circ}$ phase shifts introduced by the four wave mixing and Bragg processes (see eqs. 1, 2). Our value of $77 \pm 9^{\circ}$ agrees fairly well with this, and the small discrepancy could be due to the Bragg beams not being fully resonant. It is also interesting to note that the position of the fringe minimum is a direct signature of the positive sign of the scattering length $a$, since a negative scattering length would have resulted in a minimum at $270^{\circ}$. Usually, only the cross section, which is proportional to $a^{2}$, is measured using these momentum changing collisions.

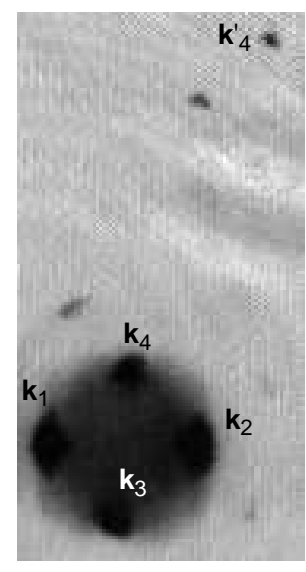

FIG. 1: The two source waves $\mathbf{k}_{1}, \mathbf{k}_{2}$, the seed $\mathbf{k}_{3}$, the conjugate wave $\mathbf{k}_{4}$, and the detection state $\mathbf{k}_{4}^{\prime}$ after $20 \mathrm{~ms}$ ballistic expansion. Also seen is the collisional halo around all four waves as well as several small unlabelled waves, generated by off-resonant scattering. The field of view is $1.0 \times 1.9 \mathrm{~mm}$.

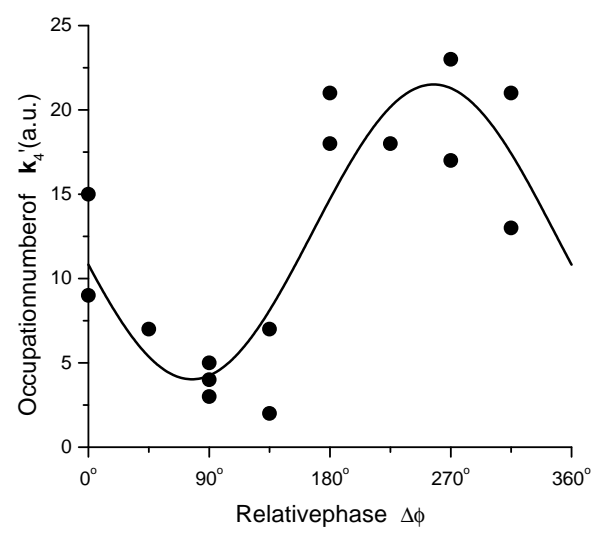

FIG. 2: Coherence of the conjugate wave. Shown is the interference of the conjugate wave with a reference wave. The relative phase shift was introduced by shifting the phase of the rf-wave which drives the lower-frequency acousto-optic modulator in the Bragg setup.

A direct implication of the sustained coherence is the reversibility of the amplification. If $\Delta \varepsilon$ is small, the second term in eq. 1 becomes negligible and the overall evolution is solely determined by the unitary four-wave mixing term. By changing the sign of one of the four waves, we can apply the inverse operator and the amplified populations will subsequently deamplify to zero due to the entanglement between the coupled modes. This is true even for the modes which were not deliberately seeded, since spontaneous emission into these modes also occurs by the same pairwise collision process. A similar experiment using photons has already been performed [11].

After $80 \mu \mathrm{s}$ of amplification, we initiated deamplification by inducing a $180^{\circ}$ phase shift in $\mathbf{k}_{2}$. The sign reversal was achieved by applying a $120 \mu \mathrm{s} \pi$-pulse resonant with the momentum difference between the source 


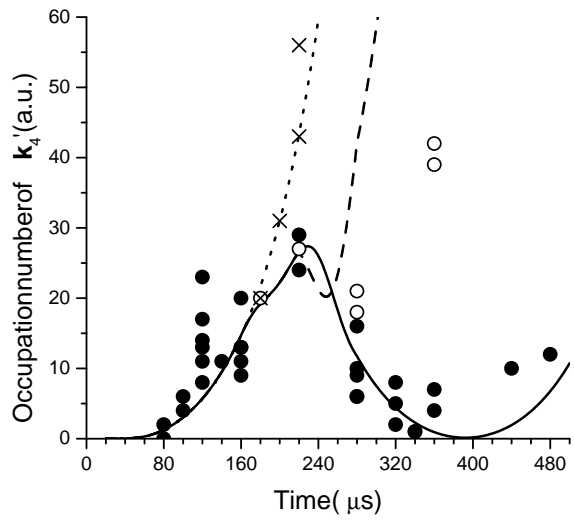

FIG. 3: Phase coherent amplification and deamplification: $(\bullet)$ Deamplification of the conjugate wave. (o) Continued amplification due to a $\pi$-pulse with a $180^{\circ}$ phase shift applied in mid-pulse (see text). ( $\times$ ) Uninterrupted amplification. The solid, dashed and dotted lines are independent numerical simulations of the respective processes.

waves $\mathbf{k}_{1}$ and $\mathbf{k}_{2}$. (For this experiment, also the $\pi / 2$-pulse which originally generated $\mathbf{k}_{2}$ was prolonged to $60 \mu \mathrm{s}$.) To check that the amplification process was not permanently disrupted by the Bragg pulse, we also performed a control experiment where the $\pi$-pulse was replaced by a $\pi / 2$ and $-\pi / 2$ pulse applied in quick succession. Like the first half of the $\pi$-pulse, the $\pi / 2$ pulse transferred all the atoms from $\mathbf{k}_{2}$ back into $\mathbf{k}_{1}$, but unlike the second half of the $\pi$-pulse, the $-\pi / 2$ pulse built up mode occupation in $\mathbf{k}_{2}$ with the original phase. If the Bragg pulses did not otherwise disturb the phase relation of the system, amplification should then resume. In all three cases, we monitored the evolution of $\mathbf{k}_{4}$ by applying the detection pulse and counting the number of atoms in $\mathbf{k}_{4}^{\prime}$ after variable intervals.

Fig. 3 shows the results obtained and clearly demonstrates the reversal of the amplification process. As a quantitive measure of the deamplification process, we defined the deamplification factor as the ratio of the maximum mode occupation to the remnant left at the end of deamplification, and obtained a factor of $\sim 5$. In addition, we infer that this reversal is phase-dependent, since amplification continues if we do not reverse the phase of the second source wave (Fig. $3(\circ, \times)$ ). This demonstrates that the four-wave mixing process does not suffer significant decoherence during the observation time.

In Fig. 3, we also plot numerical simulations of all three cases and find good qualitative agreement with the experiment. For these simulations, the energy mismatch $\Delta \varepsilon$ was the main adjustable parameter and $a_{1}$ and $a_{2}$ were taken to be c-numbers since they are highly occupied relative to the other modes. Apart from $\Delta \varepsilon$, the only other adjustable parameters in the simulations were an overall normalization constant and the size of the initial seed, which was assumed to be small. Numerically, opti- mal deamplification was achieved for $\Delta \varepsilon=h \times 1.0 \mathrm{kHz}$, and adjustments to this value by $h \times 0.6 \mathrm{kHz}$ either way still yielded a fit consistent with the experimental data. The simulations also took into account the off-resonant scattering of the seed and conjugate wave during the $\pi$ pulse, which caused a $20 \%$ loss of atoms from the amplified waves and accounted for the dip in mode occupation seen in Fig. 3(०). Finally, beyond $300 \mu$ s, amplification slows down in Fig. 3(०) and departs from the numerical simulation (dashed line) due to depletion of the source waves as a result of scattering and subsequent amplification of the unseeded modes [2].

Theoretically, maximum deamplification should occur for $\Delta \varepsilon=0$ in the absence of any extraneous phase shifts. Our experiment deviates from this ideal situation as it suffered from off-resonant scattering during the $\pi$-pulse resulting in a calculated phase shift of $0.6 \mathrm{rad}$ in the amplified modes. This was due to the finite duration of the $\pi$-pulse, which off-resonantly coupled out atoms from the amplified waves. The observed value of $\Delta \varepsilon=h \times$ $1.0 \mathrm{kHz}$ therefore might have compensated for this phase shift as the amplified modes evolved over time.

At the same time, we also discovered that the deamplification process is extremely phase-sensitive. Compared to the tolerance in $\Delta \varepsilon$ for amplification [2], the $h \times 0.6 \mathrm{kHz}$ tolerance for deamplification is relatively small. While amplification is inherently a stable process which enforces a certain phase relationship between the four waves, deamplification is unstable with respect to the phase. As soon as the phase relation is lost, deamplification ends and amplification resumes. Fortunately, deamplification remains possible since its tolerance is still larger than the estimated $h \times 0.4 \mathrm{kHz}$ halfwidth of the inhomogeneous mean field shift. However, in order to remain within the narrow range of $\Delta \varepsilon$ allowed, we had to align the Bragg beams to within milliradians of the optimal value.

In principle, the pairwise collisions of atoms during deamplification could be used to demonstrate relative number squeezing and entanglement between the two amplified modes. If decoherence is absent, the mode occupations after deamplification will reflect the relative number uncertainty in the initial waves. For coherent or classical waves, the remnant will follow Poissonian statistics and be of order $\sqrt{N}$, where $N$ is the initial mode occupation. In contrast, for perfect two mode number squeezing, the remnant will always be exactly zero. Moreover, complete deamplification of the seed and conjugate waves can only occur if the sum of their individual phases remain welldefined throughout the process. These two conditions cannot be met for unentangled waves, since their individual number and phase are canonically conjugate variables and cannot simultaneously be well-defined.

To illustrate this, we simulated the evolution of the conjugate wave for our experimental conditions, this time without an initial seed (Fig. Aa). Here, complete deam- 


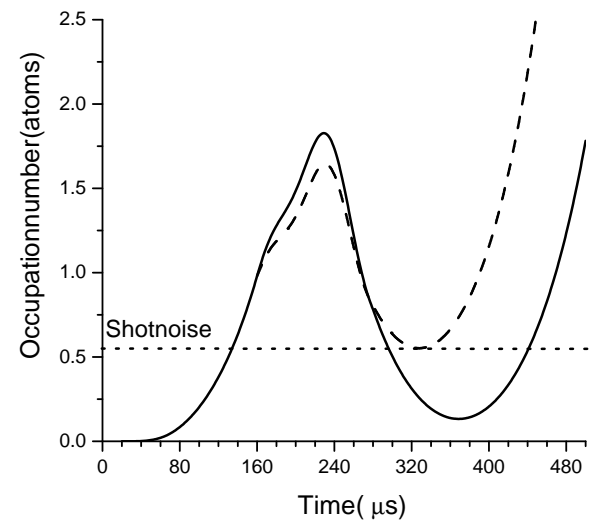

FIG. 4: Deamplification of perfectly squeezed modes with off-resonant scattering (solid line). Identical process but with coherent waves (dashed line). The vertical scale is the expectation value of the mode occupation.

plification does not occur as the squeezing has been degraded by off-resonant scattering. However, it still dips below the shot noise level given by the minimum of the control graph (Fig. Ab) which assumes coherent waves. The control simulation is started at $160 \mu \mathrm{s}$, immediately preceding the $\pi$-pulse, using non-entangled coherent waves with the same unity mode occupation. To detect relative number squeezing, we need to obtain deamplification factors of greater than $\sqrt{N}$.

Experimentally, direct detection of relative number squeezing in our strongly occupied beams is not possible since it would require a much larger deamplification factor than what we currently observe. In this, we are limited by the inhomogeneous mean field and the depletion of the source waves. However, for initially unseeded modes, a deamplification factor of five is already evidence for sub-shot noise correlations (Fig. 1 ). This shows that by monitoring a collection of modes with a similar energy mismatch, detection of the entanglement is possible with current experimental parameters. Practically, however, the occupation in these modes was masked by surrounding modes with a different energy mismatch. Further complicating detection, the momenta of the states were blurred out during the motion of the outcoupled atoms in the gradient of the mean field of the condensate.

Finally, we briefly consider other possible sources of decoherence in our amplifier and discuss how their effects may be minimized. Collisions between the source and amplified waves can compromise squeezing as atoms are scattered into or out of the amplified waves in a random way. As shown in [2], at low density, no stimulated scattering occurs and the spontaneous scattering rate was estimated to be significant only after the source waves are about to be depleted. By further manipulating the size of the condensate such that the amplified waves separate out before the source waves are depleted, we can ensure that the squeezing will be preserved.

A further study of the non-degenerate matter wave amplifier should explore the issue of the "beam quality" of the squeezed waves. The phase errors incurred due to the inhomogeneous mean field induces spatially dependent phase shifts in the wavefunction of the squeezed waves, rendering them hard to isolate. Other sources of phase errors are not a concern as they appear to have little effect on our experiment. In particular, phase fluctuations across the condensate (first observed by [8]) are absent for our parameters 12, 13. Moreover, since the atoms travel at a maximum velocity of $20 \mathrm{~mm} / \mathrm{s}$ for a duration of $300 \mu \mathrm{s}$, the required coherence length of $6 \mu \mathrm{m}$ is easily satisfied.

The collisional matter wave amplifier studied here complements the amplifier based on optical superradiance 14. 15. While the gain and interference contrast of both are comparable, the collisional amplifier has a much larger momentum bandwidth, amounting to a significant fraction of the speed of sound [2]. In contrast, the optical amplifier has a bandwidth of only a single mode 16$]$.

In conclusion, we have demonstrated the coherence of a non-degenerate matter wave amplifier by measuring the phase of the conjugate wave and by reversing the amplification process. While we do not yet directly detect number-squeezing in our amplified beams, we have evaluated all known decoherence processes and concluded that they are well controlled in our experiment. Therefore the atomic non-degenerate parametric amplifier studied here is a promising candidate for generating entangled and number squeezed beams.

We thank K. Xu and J. Abo-Shaeer for experimental assistance. We would also like to acknowledge Z. Hadzibabic, A. Chikkatur and K. Dieckmann for their valuable comments and insightful discussions. This work was funded by ONR, NSF, ARO, NASA, and the David and Lucile Packard Foundation.

* Group website: http://cua.mit.edu/ketterle_group/

[1] L. Deng, E. W. Hagley, J. Wen, M. Trippenbach, Y. Band, P. S. Julienne, J. E. Simsarian, K. Helmerson, S. L. Rolston, and W. D. Phillips, Nature 398, 218 (1999).

[2] J. M. Vogels, K. Xu, and W. Ketterle, Phys. Rev. Lett. 89, 020401 (2002).

[3] The atomic amplifier is non-degenerate in the sense that both amplified modes are distinct. This should not be confused with the energetic degeneracy of all four waves in the center-of-mass frame.

[4] M. J. Holland and K. Burnett, Phys. Rev. Lett. 71, 1355 (1993).

[5] P. Bouyer and M. A. Kasevich, Phys. Rev. A 56, R1083 (1997).

[6] M. Greiner, O. Mandel, T. W. Haensch, and I. Bloch, preprint cond/mat 0207196. 
[7] F. Dalfovo, S. Giorgini, L. P. Pitaevskii, and S. Stringari, Rev. Mod. Phys. 71, 463 (1999).

[8] S. Dettmer, D. Hellweg, P. Ryytty, J. J. Arlt, W. Ertmer, K. Sengstock, D. S. Petrov, G. V. Shlyapnikov, H. Kreutzmann, L. Santos, et al., Phys. Rev. Lett. 87, 160406 (2001).

[9] M. Kozuma, L. Deng, E. W. Hagley, J. Wen, R. Lutwak, K. Helmerson, S. L. Rolston, and W. D. Phillips, Phys. Rev. Lett. 82, 871 (1999).

[10] A. P. Chikkatur, A. Görlitz, D. M. Stamper-Kurn, S. Inouye, S. Gupta, and W. Ketterle, Phys. Rev. Lett. 85, 483 (2000).

[11] A. Lamas-Linares, J. C. Howell, and D. Bouwmeester, Nature 412, 887 (2001).
[12] M. R. Andrews, C. G. Townsend, H.-J. Miesner, D. S. Durfee, D. M. Kurn, and W. Ketterle, Science 275, 637 (1997).

[13] J. Stenger, S. Inouye, A. P. Chikkatur, D. M. StamperKurn, D. E. Pritchard, and W. Ketterle, Phys. Rev. Lett. 82, 4569 (1999).

[14] S. Inouye, T. Pfau, S. Gupta, A. P. Chikkatur, A. Görlitz, D. E. Pritchard, and W. Ketterle, Nature 402, 641 (1999).

[15] M. Kozuma, Y. Suzuki, Y. Torii, T. Sugiura, T. Kuga, E. W. Hagley, and L. Deng, Science 286, 2309 (1999).

[16] M. G. Moore and P. Meystre, Phys. Rev. Lett. 83, 5202 (1999). 